\title{
Can involved-field irradiation replace elective nodal irradiation in chemoradiotherapy for esophageal cancer? A systematic review and meta-analysis
}

This article was published in the following Dove Press journal:

OncoTargets and Therapy

II April 2017

Number of times this article has been viewed

\author{
Xiaoyue Wang ${ }^{1, *}$ \\ Chuanwang Miao 2,3,* \\ Zhen Chen ${ }^{4}$ \\ Wanhu $\mathrm{Li}^{5}$ \\ Shuanghu Yuan ${ }^{3}$ \\ jinming $\mathrm{Yu}^{3}$ \\ Xudong $\mathrm{Hu}^{3}$ \\ 'Department of Surgery, Shandong \\ Cancer Hospital Affiliated to \\ Shandong University, ${ }^{2}$ Department of \\ Oncology, School of Medicine and Life \\ Sciences, University of Jinan-Shandong \\ Academy of Medical Sciences, \\ ${ }^{3}$ Department of Radiation Oncology, \\ ${ }^{4}$ Department of Medical Oncology, \\ ${ }^{5}$ Department of Radiology, Shandong \\ Cancer Hospital Affiliated to \\ Shandong University, Jinan, Shandong \\ Province, People's Republic of China \\ *These authors contributed equally \\ to this work
}

Correspondence: Xudong Hu Department of Radiation Oncology, Shandong Cancer Hospital Affiliated to Shandong University, No 440, Jiyan Road, Jinan 2501 17, Shandong Province, People's Republic of China

$\mathrm{Tel}+8653$ I 867626932

Fax +86 53186762 6931

Email drhuxudong@163.com

\begin{abstract}
Chemoradiotherapy is the most common treatment for inoperable esophageal cancer. However, there is no consensus on the delineation of the clinical target volume. Elective nodal irradiation (ENI) is recommended for inoperable esophageal cancer. A few studies have reported a decrease in the incidence of radiation-related toxicity of involved-field irradiation (IFI) for esophageal cancer. A systematic review and pooled analysis were performed to determine whether IFI in definitive chemoradiotherapy was more beneficial than ENI for esophageal cancer. The results showed no significant differences in the overall survival and local control rates between the IFI and ENI arms. Meanwhile, the incidences of esophageal and lung toxicities were significantly decreased in the IFI arm. These results suggest that IFI is a feasible treatment option for locally advanced esophageal cancer, especially to minimize irradiation-related toxicity.
\end{abstract}

Keywords: chemoradiotherapy, esophageal cancer, involved-field irradiation, elective nodal irradiation, meta-analysis

\section{Introduction}

Globally, esophageal cancer is the sixth and ninth most common cause of cancer death in males and females, respectively, with the highest rates occurring in eastern Asia and in eastern and southern Africa. ${ }^{1}$ Surgical resection is recommended for the treatment of early stage esophageal cancer. However, most patients are diagnosed with advanced stage disease and are unfit for surgical resection. ${ }^{2}$ According to the results of two clinical trials performed by the Radiation Therapy Oncology Group (RTOG; RTOG85- $01^{3}$ and INT $0123^{4}$ ), definitive chemoradiotherapy is the standard treatment for patients with locally advanced esophageal cancer. However, the outcome of definitive chemoradiotherapy for advanced esophageal cancer is unsatisfactory, with a 5 -year overall survival (OS) rate of $\sim 10 \% .^{5}$

Optimal delineation of the radiotherapeutic target volume is very important to improve the therapeutic effect and decrease radiative toxicity. ${ }^{6}$ However, there is no consensus on methods to delineate the clinical target volume (CTV). The National Comprehensive Cancer Network (NCCN) guidelines (version 1.2016) recommend that the CTV should include the areas at risk for microscopic disease and elective nodal regions. Elective treatment of nodal regions is dependent on the location of the primary tumor in the esophagus. Although involved-field irradiation (IFI) is performed for most cases of non-small cell lung cancer and Hodgkin's lymphoma, relatively few studies have reported the results of IFI for esophageal cancer. ${ }^{7,8}$ Of these, Zhao et $\mathrm{al}^{9}$ 
reported the outcomes of radiotherapy for 53 patients with squamous cell carcinoma (SCC) of the esophagus, in which only the primary tumor and positive lymph nodes were irradiated. The results of that study showed that in-field recurrence developed in $44 \%$ of patients, distant metastasis with or without regional failure occurred in $46 \%$ of patients, and isolated out-of-field nodal recurrence developed in only $8 \%$ of patients. Other studies reported similar results. ${ }^{10,11}$ Together, these results confirmed the feasibility of IFI for locally advanced esophageal cancer.

Because of the progress of technology in radiation, it has already become a reality to irradiate the regional lymph node precisely in clinical practice. The volume of elective nodal irradiation (ENI) is greater than the volume of IFI in thoracic radiotherapy. In theory, the large radiation volume can increase side effects of radiation therapy. There were lots of life-threatening side effects in RTOG 85-01. ${ }^{3}$ On the other hand, IFI may increase the risk of nodal failure in unirradiated nodal stations, especially as the pattern of lymph node metastasis is controversial in esophageal cancer. However, no large, prospective, randomized, controlled trial has compared ENI to IFI for the treatment of esophageal cancer. The purpose of this systematic review and pooled analysis was to investigate whether ENI in definitive radiotherapy is more beneficial than IFI in a large group of patients with esophageal cancer worldwide.

In this review, we summarized the available data and concluded that IFI could be a feasible selection for locally advanced esophageal cancer, especially to minimize the risk of irradiation-related toxicity. Hence, patients who are suffering from esophageal cancer, especially elderly patients, tend to tolerate IFI better than ENI.

\section{Materials and methods Literature search}

The medical databases PubMed (https://www.ncbi.nlm. nih.gov/pubmed), Medline (https://www.medline.com/), ISI Web of Science (https://www.webofknowledge.com/), and ClinicalTrials.gov (https://ClinicalTrials.gov/) were searched for English and non-English publications (last update: May 30, 2016). The abstract database of annual meetings (American Society for Radiation Oncology, American Society of Clinical Oncology, etc) and the references of published systematic reviews were also searched for reports of IFI or ENI radiotherapy for esophageal cancer. An algorithm with the following terms was used to search the databases: 1) "esophageal OR esophagus OR esophageal"; 2) “cancer OR carcinoma OR neoplasm"; 3) "radiotherapy
OR chemoradiation OR irradiation"; 4) "IFI"; and 5) "ENI" both as text and Medical Subject Heading terms. Although no language restrictions were initially applied, the full-text review was limited to reports published in English. Additional studies were manually searched using the references cited in the selected articles.

\section{Literature selection}

The included studies were selected on the basis of the following criteria: 1) patients were histopathologically diagnosed with esophageal SCC and adenocarcinoma; 2) IFI was compared with ENI; 3) outcomes, such as OS rate, rate of acute and late toxic effects, rate of recurrence, and distant metastasis, were evaluated; and 4) $\geq 10$ cases were included. Unpublished data, case reports, reviews, letters, editorials, comments, and studies that applied either IFI or ENI alone were excluded from the analysis. Multiple articles published by the same author over a short period were also excluded.

\section{Data extraction and statistical analysis}

The abstract of each retrieved article was reviewed using the keywords. Irrelevant citations were removed in accordance with the criteria mentioned in the "Literature selection" section. Two reviewers independently evaluated the full text of each article and extracted information regarding the methods and results.

All statistical analyses were performed using the Review Manager (version 5.3) software package (http://tech.cochrane. org/revman). Heterogeneity among the different studies was examined using the chi-squared test. A probability $(P)$ value of $<0.05$ was considered as statistically significant. Different models were adopted to calculate the diagnostic indexes according to heterogeneity. A fixed-effect model was used when $P$ was $>0.05$, and a random-effect model was used when $P$ was $<0.05$.

\section{Results}

\section{Characteristics of selected articles}

The electronic search identified 638 potentially relevant publications according to the defined criteria. After examination of the abstracts and full-text articles by the reviewers, 634 articles were excluded, whereas four articles met all the inclusion criteria. In addition, an abstract from the 2015 American Society for Radiation Oncology (ASTRO) annual meeting was included after a manual search. The study selection process is summarized in Figure 1.

Finally, five studies with a total of 757 patients with esophageal cancer were included in the meta-analysis. The 


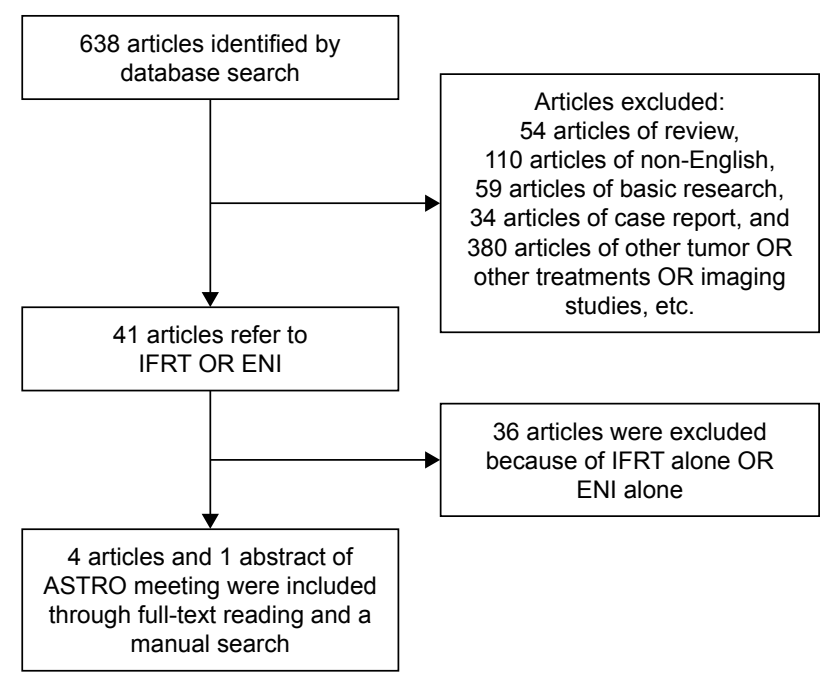

Figure I Selection criteria of the included studies.

Abbreviations: IFRT, involved-field radiotherapy; ENI, elective nodal irradiation; ASTRO, American Society for Radiation Oncology.

histology of most patients was SCC. Three-dimensional conformal radiotherapy and conventional radiotherapy were most often applied. Of the 757 patients, 406 (53.6\%) received IFI and 351 (46.4\%) received ENI. The basic characteristics of the included studies are shown in Table 1.

\section{OS rates}

Three studies reported 1-year, 2-year, and 3-year OS rates; one study only reported 1- and 2-year OS rates; and one study only reported 3-year OS rates. ${ }^{12-16}$ These OS outcomes were pooled for analysis with Review Manager (version 5.3) software. The results showed that the heterogeneities of the 1-year, 2-year, and 3-year OS outcomes were $P=0.58, P=0.1$, and $P=0.1$, respectively, which indicated that there was no heterogeneity across the included studies. The relative risk ratio (RR) value, expressed as IFI versus ENI, was 1.0 (95\% confidence interval $[\mathrm{CI}]=0.92-1.08, P=0.94)$ for the 1-year survival rate, 1.04 $(95 \% \mathrm{CI}=0.90-1.19, P=0.61)$ for the 2-year survival rate, and $1.11(95 \% \mathrm{CI}=0.92-1.35, P=0.27)$ for the 3 -year survival rate. These results indicate no difference in the 1-year, 2-year, and 3 -year OS rates between the IFI and ENI arms.

The median OS duration was reported in three studies. ${ }^{12-14}$ As shown in Figure 2, there was no significant difference in the pooled median OS duration between the IFI and ENI arms (15.5-38.9 months vs 17-32.7 months, respectively; pooled $\mathrm{RR}$ value $=1.2 ; 95 \% \mathrm{CI}=0.75-1.91 ; P=0.44)$.

\section{Local control rates (LCRs) and patterns of treatment failure}

The 1-year, 2-year, and 3-year LCRs were reported in three studies. ${ }^{12-14}$ There was no heterogeneity in 1 -year $(P=0.05)$

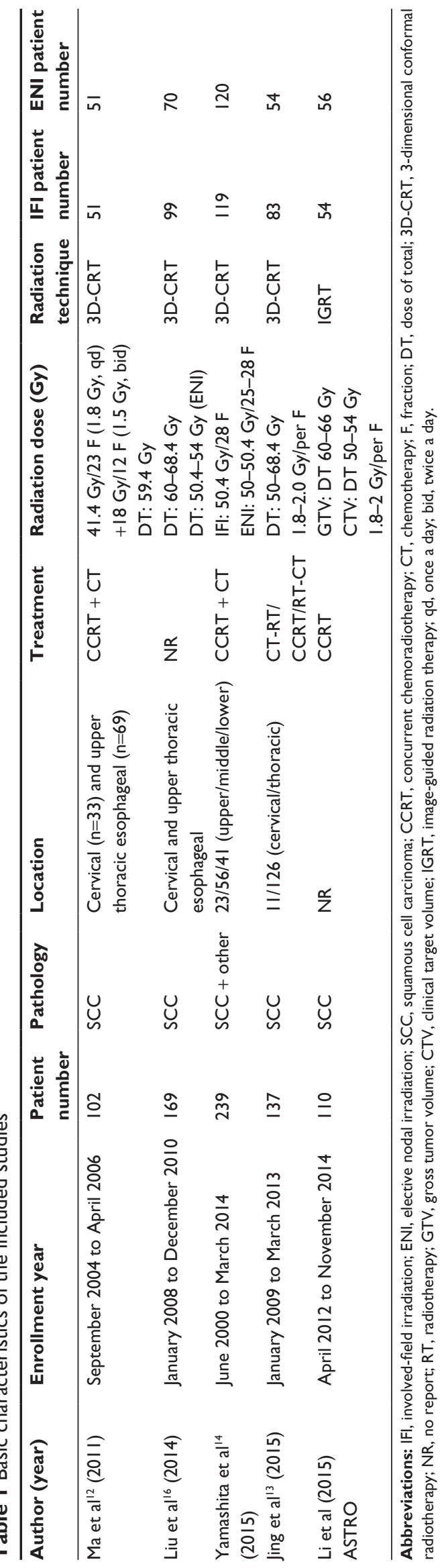




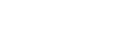

\section{1-year OS for enrolled studies}

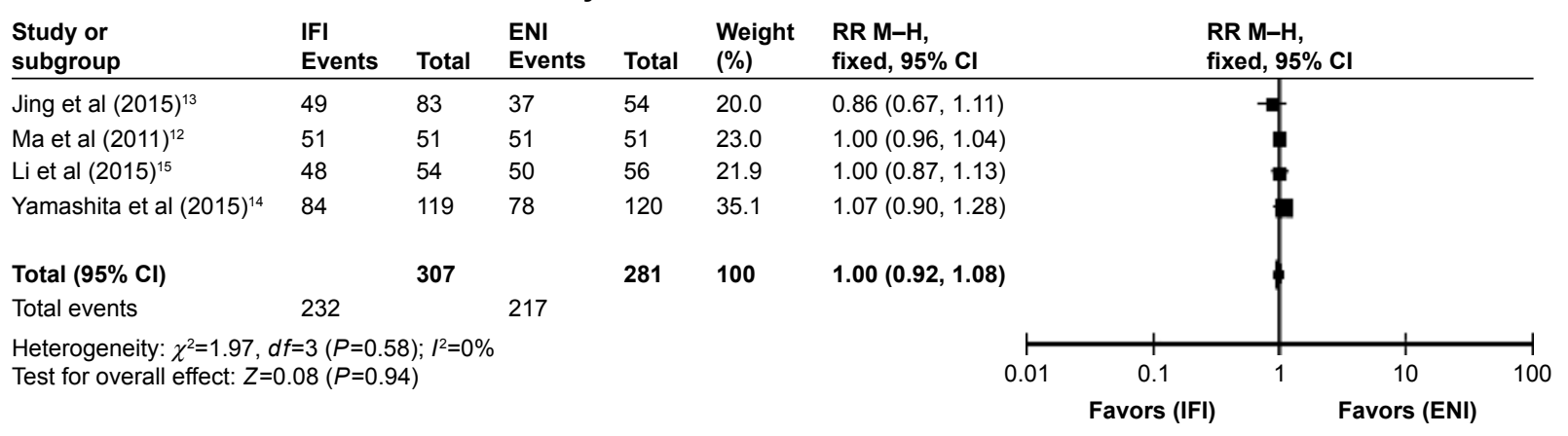

\section{2-year OS for enrolled studies}

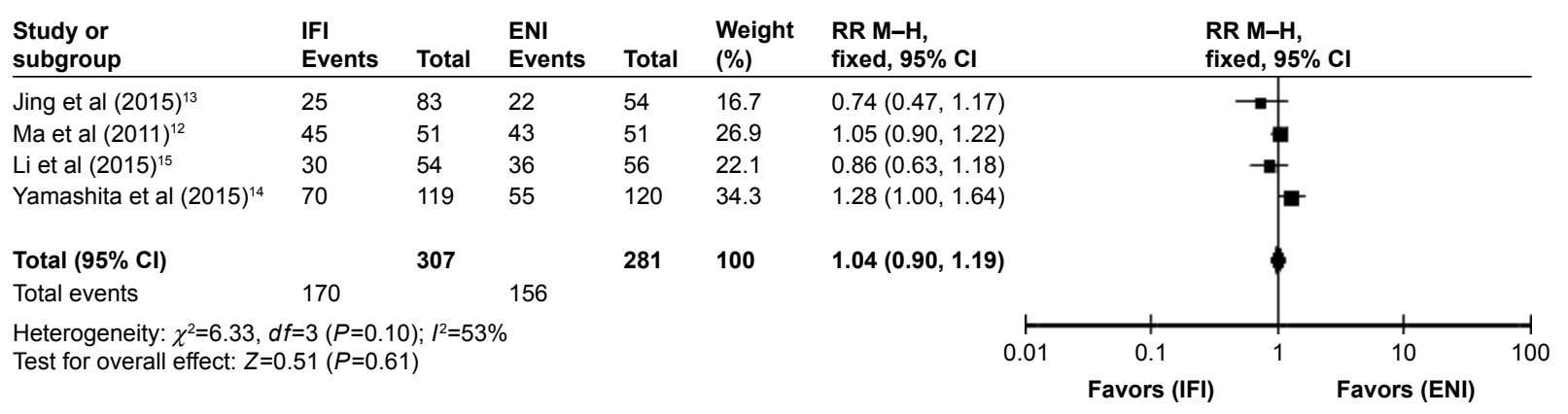

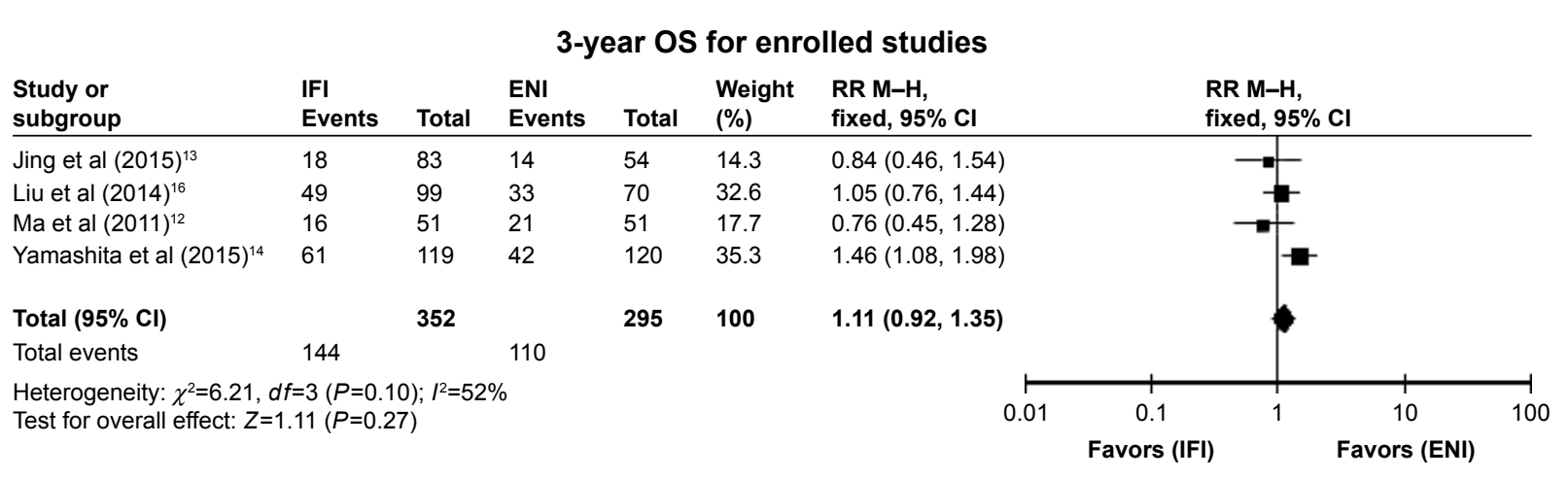

\section{Median survival time for enrolled studies}

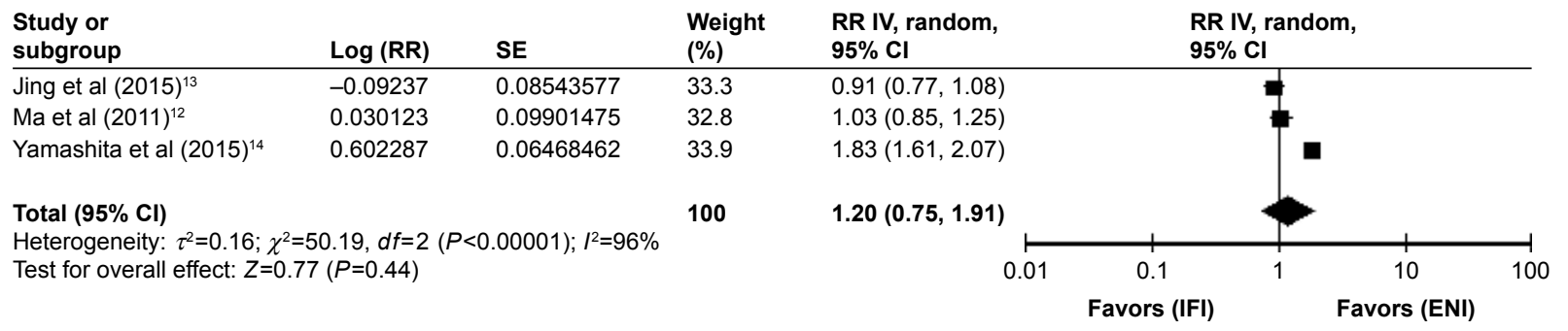

Figure 2 Forest plots comparing OS and median OS time outcomes for IFI and ENI.

Abbreviations: OS, overall survival; IFI, involved-field irradiation; ENI, elective nodal irradiation; RR, risk ratio; $\mathrm{M}-\mathrm{H}$, Mantel-Haenszel; $\mathrm{Cl}$, confidence interval; SE, standard error; IV, inverse variance.

and 3-year $(P=0.14)$ LCRs across the included studies. Thus, the fixed-effect model was selected for the pooled analysis. The random-effect model was selected for the 2-year LCR analysis, with statistical tests of heterogeneity showing $P=0.03$. The results of pooled analysis showed no significant difference between the IFI and ENI arms in the 1-year
LCR $(\mathrm{RR}=1.07,95 \% \mathrm{CI}=0.94-1.20, P=0.30), 2$-year LCR $(\mathrm{RR}=0.93,95 \% \mathrm{CI}=0.70-1.23, P=0.61)$, and 3-year LCR $(\mathrm{RR}=1.09,95 \% \mathrm{CI}=0.92-1.28, P=0.33$; Figure 3$)$.

Four studies investigated the occurrence of treatment failure, including local/regional recurrence and distant metastasis. The meta-analysis results showed no significant difference 


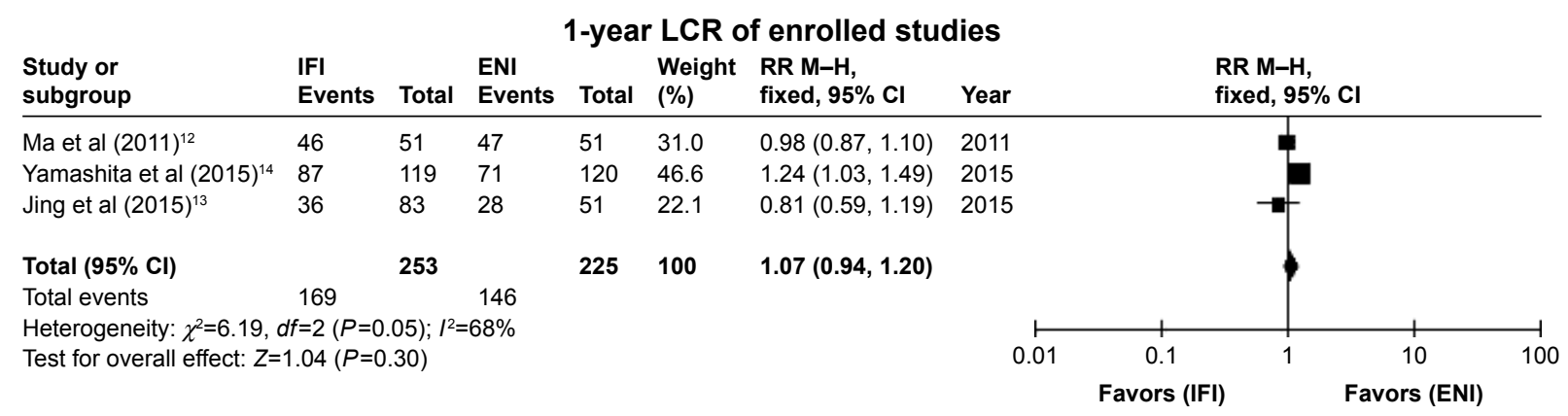

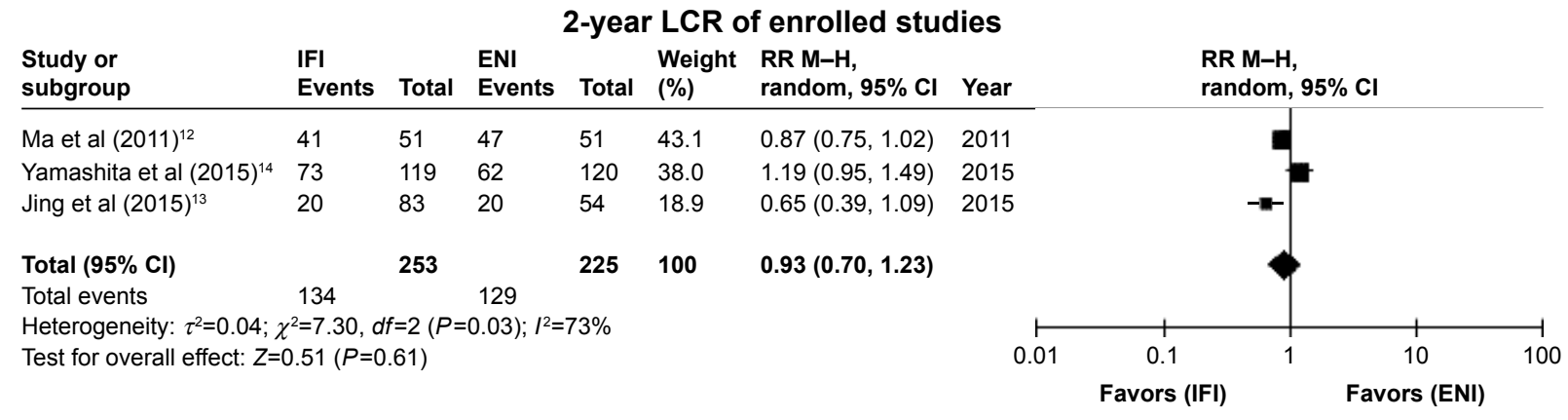

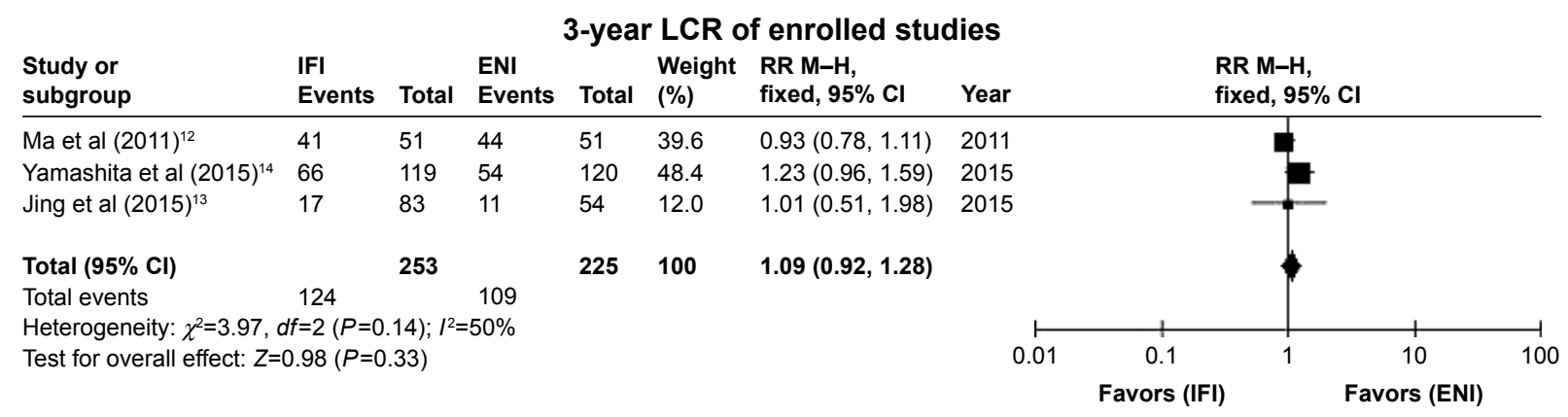

Figure 3 Forest plots comparing LCR outcomes for IFI and ENI.

Abbreviations: LCR, local control rate; IFI, involved-field irradiation; ENI, elective nodal irradiation; RR, risk ratio; $\mathrm{M}-\mathrm{H}, \mathrm{Mantel}-\mathrm{Haenszel}$; Cl, confidence interval.

in local/regional recurrence during follow-up between the IFI and ENI arms ( $\mathrm{RR}=0.93,95 \% \mathrm{CI}=0.72-1.2, P=0.59)$. However, the incidence of distant metastasis was lower among patients who received IFI radiotherapy $(\mathrm{RR}=0.70$, $95 \% \mathrm{CI}=0.51-0.94, P=0.02)$, with no heterogeneity $\left(I^{2}=33 \%\right.$, $P=0.02)$. These results indicate a lower rate of distant metastasis following IFI, as compared to ENI. The forest plot results are shown in Figure 4.

\section{Treatment-related toxicities}

The incidences of $\geq$ grade 3 acute and late treatment-related toxicities were reported in four studies, and the incidence of $\geq$ grade 2 toxicity was reported in one study. ${ }^{12-16}$ The incidences of lung-related and esophagus-related toxicities were analyzed, whereas other toxicities were not included because of incomplete data or rarity. In this meta-analysis, the incidences of acute and late lung toxicities were lower in the IFI arm than in the ENI arm, with RR values of 0.58
$(95 \% \mathrm{CI}=0.36-0.94, P=0.03)$ and $0.21(95 \% \mathrm{CI}=0.05-0.81$, $P=0.02)$, respectively. The pooled incidence of acute esophagus toxicity was significantly lower in the IFI arm than in the ENI arm ( $\mathrm{RR}$ value $=0.50,95 \% \mathrm{CI}=0.35-0.71$, $P<0.0001)$. However, there was no statistical difference in the incidence of late esophagus toxicity between the two arms ( $\mathrm{RR}$ value $=0.93,95 \% \mathrm{CI}=0.38-2.26, P=0.87$ ). The forest plot results are shown in Figure 5.

\section{Discussion}

Radiotherapy plays an important role in the treatment of inoperable esophageal cancer. The NCCN, RTOG, and European guidelines provide recommendations for radiotherapy planning, including radiation dose and target volume. However, the extent of the irradiation field remains controversial, especially for the CTV. The CTV of a primary tumor is commonly defined as $3-5 \mathrm{~cm}$ superior and inferior expansion along the length of the esophagus and a $1-1.5 \mathrm{~cm}$ 


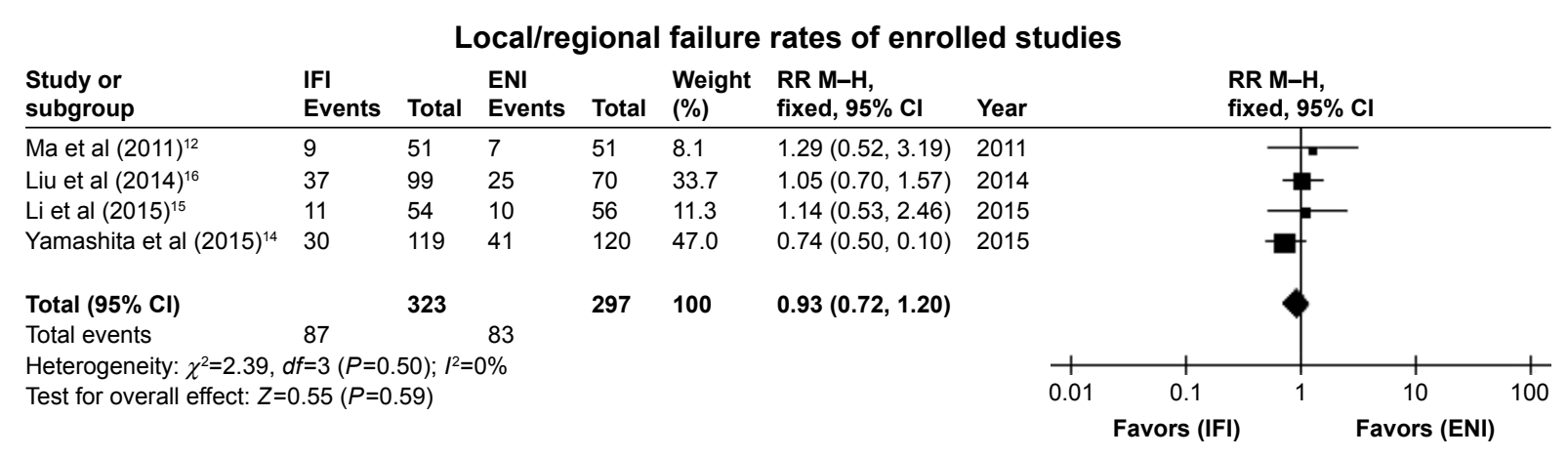

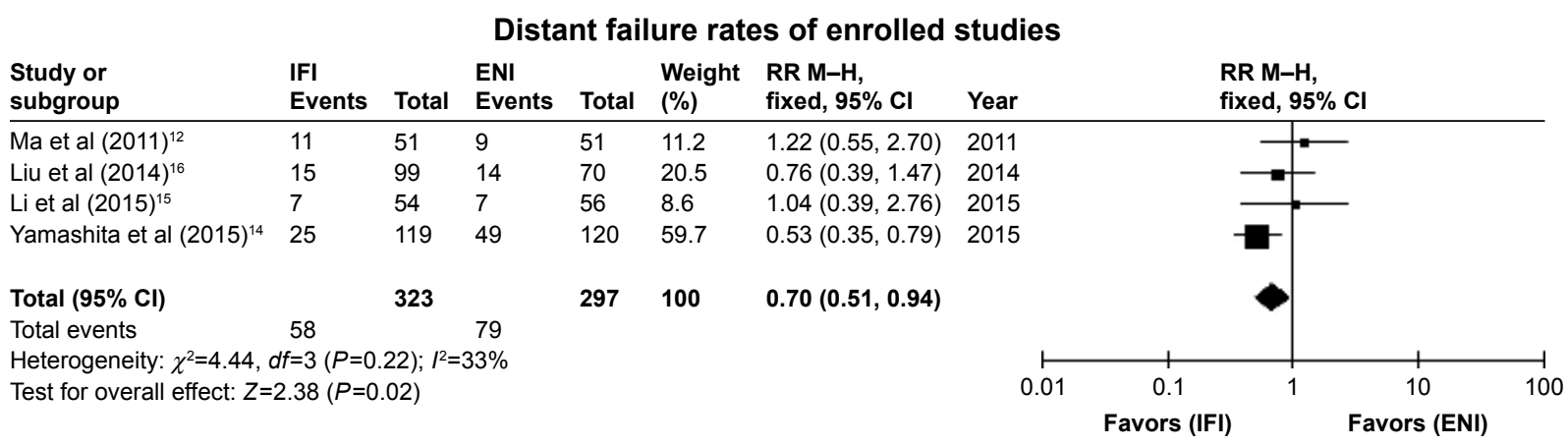

Figure 4 Forest plots comparing patterns of treatment failure for IFI and ENI.

Abbreviations: IFI, involved-field irradiation; ENI, elective nodal irradiation; RR, risk ratio; $\mathrm{M}-\mathrm{H}$, Mantel-Haenszel; $\mathrm{Cl}$, confidence interval.

radial expansion. ${ }^{3,4,17}$ At present, there is no consensus on the range of lymph node CTV. In the RTOG 85-01 trial, the range of the lymph node CTV was extended from the supraclavicular region to the gastroesophageal junction. ${ }^{3,4}$ This lymph node CTV is the target of ENI. Since then, a number of clinical studies have used ENI to target the lymph node CTV.${ }^{17} \mathrm{~A}$ few recent studies have used IFI in definitive and preoperative chemoradiotherapy for locally advanced esophageal cancer. ${ }^{9,12,18}$ Most of these studies reported no significant differences in OS or LCRs with the adopted ENI. To a certain extent, these results showed the feasibility of IFI radiotherapy for esophageal cancer. However, some oncologists claim that IFI may increase the risk of nodal failure in nonirradiated nodal stations. So far, no large, randomized, controlled, clinical trial has compared IFI to ENI.

In this meta-analysis, five studies with a total of 757 patients were pooled to investigate the feasibility of replacing IFI with ENI for esophageal cancer. The results of the present study showed no significant differences in the OS rate and median OS duration between the IFI and ENI groups. Zhao et $\mathrm{al}^{9}$ reported 1-year, 3-year, and 5-year OS rates of $77 \%, 56 \%$, and $41 \%$, respectively, after IFI radiotherapy. In a study of IFI radiotherapy for stage I esophageal cancer, Kawaguchi et al ${ }^{18}$ reported 3-year OS and disease-free survival rates of $76 \%$ and $66 \%$, respectively. A retrospective study of IFI in patients with locally advanced esophageal cancer by Zhang et al ${ }^{11}$ reported 1-year, 2-year, and 3-year OS rates of $86.3 \%, 30.0 \%$, and $18.8 \%$, respectively. A meta-analysis of ENI radiotherapy for esophageal cancer reported 1-year, 2-year, 3-year, and 5-year pooled OS rates of $57 \%, 39 \%, 40 \%$, and $29 \%$, respectively. ${ }^{19}$ The results of these studies showed no significant decrease in OS with IFI radiotherapy for esophageal cancer. Therefore, IFI radiotherapy should be considered a feasible treatment option for esophageal cancer.

The main reason that oncologists advocate the use of ENI is that the use of IFI can lead to greater regional lymph node failure. According to the results of the RTOG 94-05 trial, the rate of regional nodal failure with ENI was only $7 \%$ in both the high-dose and standard-dose arms. ${ }^{4}$ In another study, Onozawa et $\mathrm{al}^{20}$ investigated the efficacy of ENI as definitive chemoradiotherapy for esophageal cancer and found that the treatment failed in 20 patients, whereas 60 patients achieved a complete response after initial chemoradiotherapy. The local failure and distant metastasis rates were $50 \%(10 / 20)$ and $45 \%(9 / 20)$ among the 20 cases of treatment failure, and only one patient experienced elective nodal failure. A retrospective study by $\mathrm{Li}$ et $\mathrm{al}^{21}$ reported infield recurrence, distant metastasis, and out-of-field regional failure rates of $69.6 \%, 33.9 \%$, and $12.5 \%$, respectively, in 56 patients with T4 esophageal SCC who received IFI treatment. In the present meta-analysis, there was no significant 


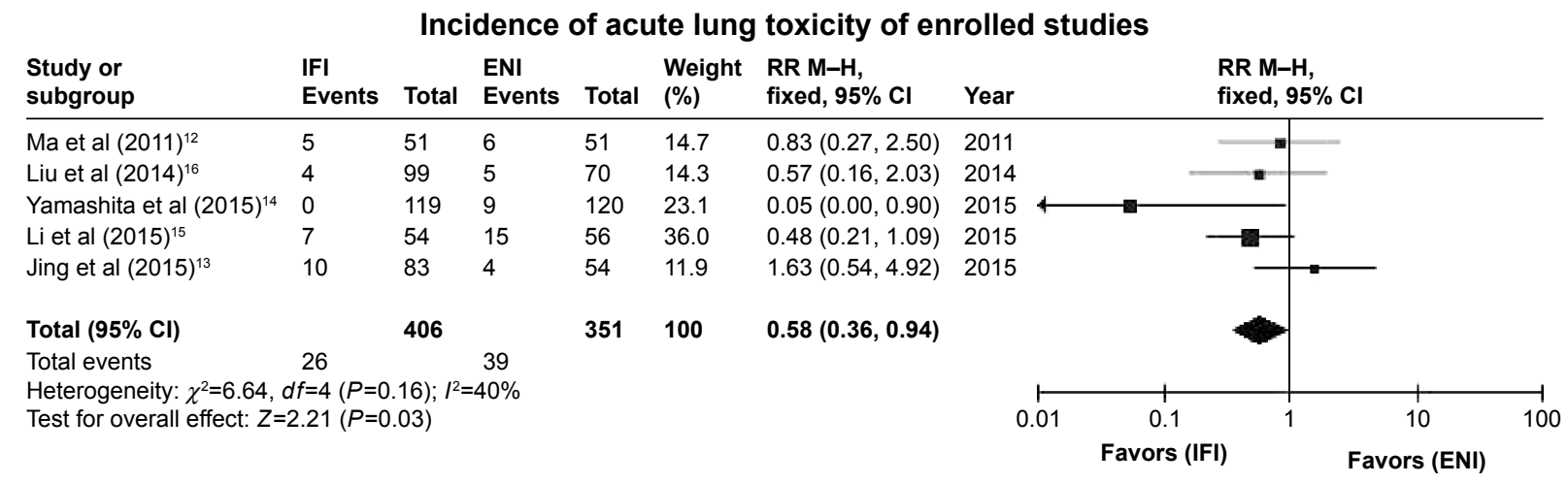

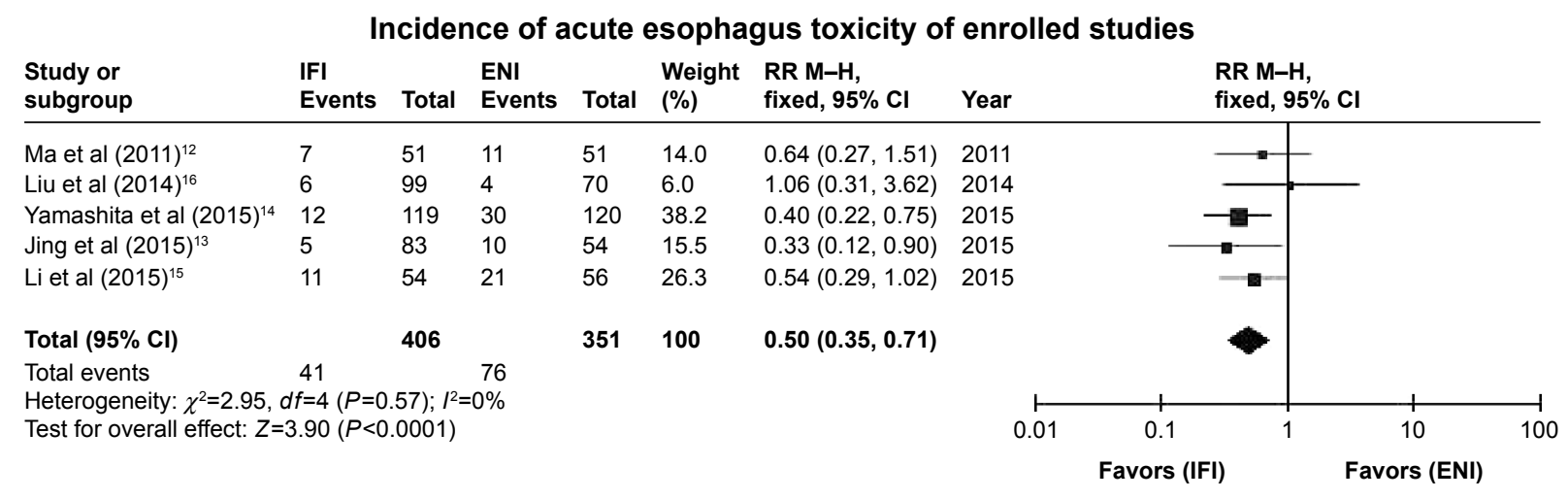

Incidence of late lung toxicity of enrolled studies

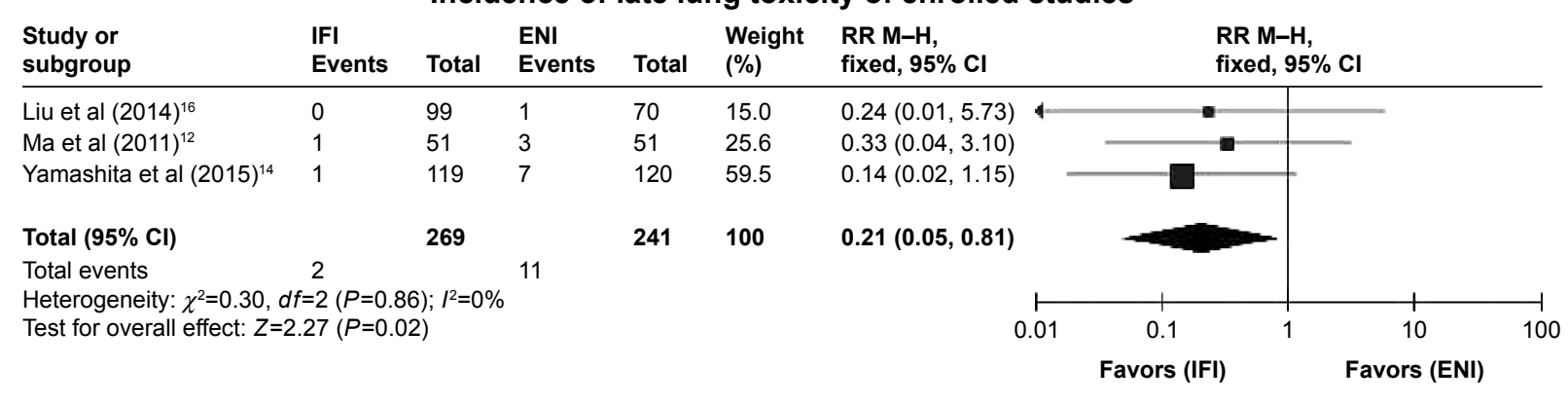

Incidence of late esophagus toxicity of enrolled studies

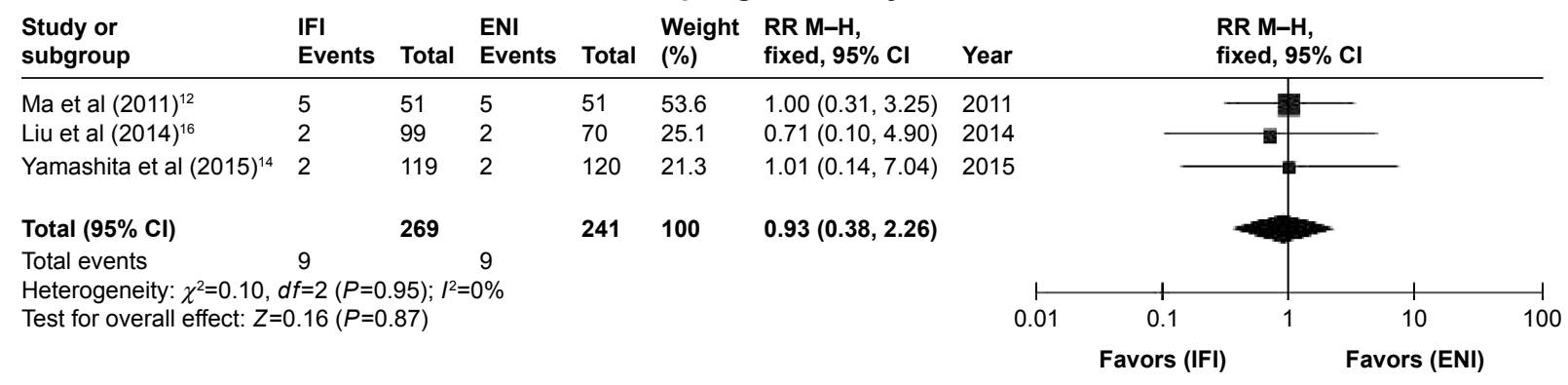

Figure 5 Forest plots comparing treatment-related toxicities for IFI and ENI.

Abbreviations: IFI, involved-field irradiation; $\mathrm{ENI}$, elective nodal irradiation; RR, risk ratio; $\mathrm{M}-\mathrm{H}$, Mantel-Haenszel; $\mathrm{Cl}$, confidence interval.

difference in pooled local/regional recurrence between the IFI and ENI arms. Moreover, statistical analysis showed a lower incidence of distant metastasis following IFI radiotherapy, which was possibly because of a greater weight of the results of one of the included studies. In this particular study, the number of patients given two or more cycles of chemotherapy was higher in the IFI arm (104/119) that in the ENI arm (92/120). ${ }^{14}$ More cycles of chemotherapy may explain 
the lower incidence of distant metastasis. Nevertheless, the results of the present study and previous studies showed that the regional nodal failure rate of IFI was acceptable in esophageal cancer patients.

In theory, a smaller range of CTV can decrease irradiationrelated toxicity. The results of the present study showed that the incidences of acute and late lung toxicities and acute esophagus toxicity were lower in the IFI arm than in the ENI arm. However, there was no significant difference in the incidence of late esophagus toxicity between the two arms, possibly because the dose to the gross tumor volume was similar between the two arms and the dose to the esophagus included in the region of elective nodes was usually relatively low. The incidences of $\geq$ grade 3 acute and late radiation toxicities were $71 \%$ and $34 \%$, respectively, in the standarddose arm (50.4 Gy) of the RTOG 94-05 trial. ${ }^{4}$ Meanwhile, the rates of treatment-related death were $10 \%$ (11/109) in the high-dose arm (64.8 Gy) and 2\% (2/109) in the standard-dose arm. Zhao et $\mathrm{al}^{9}$ reported incidences of grade 3 acute and late toxicities of $9 \%$ and $6 \%$, respectively, using IFI radiotherapy, with no acute or late grade 4 or 5 toxicity. In summary, the rate of radiation-related toxicity was significantly lower with IFI than with ENI radiotherapy. Furthermore, severe acute and late radiation toxicity increases patient mortality, thus decreasing the benefit of treatment.

There were several limitations to this review. First, only five studies were included and all patients were Asian. Second, the most common tumor histology was esophageal SCC. Third, there was a potential risk of bias in patient selection (including age, gender, and tumor location) and heterogeneity in radiation technique and dose, which might have influenced the pooled results. Therefore, the accuracy of our conclusion is affected by these limitations and it needs further studies with large, multicenter, randomized clinical trials to verify.

\section{Conclusion}

The results of this meta-analysis showed that there were no significant differences in OS and LCRs between the IFI and ENI arms. Meanwhile, the incidences of esophageal and lung toxicities were significantly decreased in the IFI arm. These results suggest that IFI could be a feasible selection for locally advanced esophageal cancer, especially to minimize the risk of irradiation-related toxicity. Future multicenter prospective randomized clinical trials are needed to verify these results and to determine whether IFI is a better choice than ENI for the treatment of esophageal cancer.

\section{Disclosure}

The authors report no conflicts of interest in this work.

\section{References}

1. Torre LA, Bray F, Siegel RL, Ferlay J, Lortet-Tieulent J, Jemal A. Global cancer statistics, 2012. CA Cancer J Clin. 2015;65(2):87-108.

2. Paul S, Altorki N. Outcomes in the management of esophageal cancer. J Surg Oncol. 2014;110(5):599-610.

3. Cooper JS, Guo MD, Herskovic A, et al. Chemoradiotherapy of locally advanced esophageal cancer: long-term follow-up of a prospective randomized trial (RTOG 85-01). Radiation Therapy Oncology Group. JAMA. 1999;281(17):1623-1627.

4. Minsky BD, Pajak TF, Ginsberg RJ, et al. INT 0123 (Radiation Therapy Oncology Group 94-05) phase III trial of combined-modality therapy for esophageal cancer: high-dose versus standard-dose radiation therapy. J Clin Oncol. 2002;20(5):1167-1174.

5. Dai T, Shah MA. Chemoradiation in oesophageal cancer. Best Pract Res Clin Gastroenterol. 2015;29(1):193-209.

6. Fokas E, Rödel C. Definitive, preoperative, and palliative radiation therapy of esophageal cancer. Viszeralmedizin. 2015;31(5):347-353.

7. Cheung PC, Mackillop WJ, Dixon P, Brundage MD, Youssef YM, Zhou S. Involved-field radiotherapy alone for early-stage non-small-cell lung cancer. Int J Radiat Oncol Biol Phys. 2000;48(3):703-710.

8. Ansell SM. Hodgkin lymphoma: diagnosis and treatment. Mayo Clin Proc. 2015;90(11):1574-1583.

9. Zhao KL, Ma JB, Liu G, Wu KL, Shi XH, Jiang GL. Three-dimensional conformal radiation therapy for esophageal squamous cell carcinoma: is elective nodal irradiation necessary. Int J Radiat Oncol Biol Phys. 2010; 76(2):446-451.

10. Sanuki N, Ishikura S, Shinoda M, Ito Y, Hayakawa K, Ando N. Radiotherapy quality assurance review for a multi-center randomized trial of locally advanced esophageal cancer: the Japan Clinical Oncology Group (JCOG) trial 0303. Int J Clin Oncol. 2012;17(2):105-111.

11. Zhang X, Li M, Meng X, et al. Involved-field irradiation in definitive chemoradiotherapy for locally advanced esophageal squamous cell carcinoma. Radiat Oncol. 2014;9:64.

12. Ma JB, Song YP, Yu JM, et al. Feasibility of involved-field conformal radiotherapy for cervical and upper-thoracic esophageal cancer. Onkologie. 2011;34(11):599-604.

13. Jing W, Zhu H, Guo H, et al. Feasibility of elective nodal irradiation (ENI) and involved field irradiation (IFI) in radiotherapy for the elderly patients (aged $\geq 70$ years) with esophageal squamous cell cancer: a retrospective analysis from a single institute. PLoS One. 2015;10(12): e0143007.

14. Yamashita H, Takenaka R, Omori M, et al. Involved-field radiotherapy (IFRT) versus elective nodal irradiation (ENI) in combination with concurrent chemotherapy for 239 esophageal cancers: a single institutional retrospective study. Radiat Oncol. 2015;10:171.

15. Li T, Yisikandaer A, Zhang X, et al. Involved-field irradiation vs elective nodal irradiation for locally advanced thoracic esophageal squamous cell carcinoma: a comparative interim analysis of clinical outcomes and toxicities (NCT01551589, CSWOG 003). Int J Radiat Oncol Biol Phys. 2015;93(3S suppl):S3.

16. Liu M, Zhao K, Chen Y, Jiang GL. Evaluation of the value of ENI in radiotherapy for cervical and upper thoracic esophageal cancer: a retrospective analysis. Radiat Oncol. 2014;9:232.

17. Yamashita H, Okuma K, Wakui R, Kobayashi-Shibata S, Ohtomo K, Nakagawa K. Details of recurrence sites after elective nodal irradiation (ENI) using 3D-conformal radiotherapy (3D-CRT) combined with chemotherapy for thoracic esophageal squamous cell carcinoma a retrospective analysis. Radiother Oncol. 2011;98(2):255-260.

18. Kawaguchi Y, Nishiyama K, Miyagi K, Suzuki O, Ito Y, Nakamura S. Patterns of failure associated with involved field radiotherapy in patients with clinical stage I thoracic esophageal cancer. Jpn J Clin Oncol. 2011; 41(8):1007-1012. 
19. Du D, Song T, Liang X, Fang M, Wu S. Concurrent chemoradiotherapy with elective lymph node irradiation for esophageal cancer: a systemic review and pooled analysis of the literature. Dis Esophagus. Epub 2016 Feb 26.

20. Onozawa M, Nihei K, Ishikura S, et al. Elective nodal irradiation (ENI) in definitive chemoradiotherapy (CRT) for squamous cell carcinoma of the thoracic esophagus. Radiother Oncol. 2009;92(2):266-269.
21. Li M, Zhao F, Zhang X, et al. Involved-field irradiation in definitive chemoradiotherapy for T4 squamous cell carcinoma of the esophagus. Curr Oncol. 2016;23(2):e131-e137.

\section{Publish your work in this journal}

OncoTargets and Therapy is an international, peer-reviewed, open access journal focusing on the pathological basis of all cancers, potential targets for therapy and treatment protocols employed to improve the management of cancer patients. The journal also focuses on the impact of management programs and new therapeutic agents and protocols on

\section{Dovepress}

patient perspectives such as quality of life, adherence and satisfaction. The manuscript management system is completely online and includes a very quick and fair peer-review system, which is all easy to use. Visit http://www.dovepress.com/testimonials.php to read real quotes from published authors.

Submit your manuscript here: http://www.dovepress.com/oncotargets-and-therapy-journal 